\title{
Global burden of diabetes: regional disparities in prevalence, incidence, and mortality
}

\author{
Maria Bota ${ }^{1,2}$, Alice Koechlin ${ }^{1,2}$, Cécile Pizot ${ }^{1}$, Miruna Dragomir ${ }^{1}$, Alina Macacu ${ }^{1}$, Gilles Guillot ${ }^{1}$, Peter Boyle ${ }^{1,2}$ \\ ${ }^{1}$ International Prevention Research Institute (iPRI), Lyon, France \\ 2Strathclyde University Global Public Health Institute, Lyon, France
}

\begin{abstract}
Objectives: To study the trends in diabetes prevalence, incidence, and mortality over time and by region of the world.

Material and methods: An online literature search was carried out in PubMed for studies reporting data on the time trends of the prevalence and of the incidence of diabetes. Studies reporting the most recent trends were selected to represent each region of the world and with the aim to cover a time span as long as possible. For mortality trends, diabetes deaths registered in the World Health Organisation (WHO) mortality database by November 2018 were extracted by gender and five-year age group for a selection of countries and for the longest period available (from 1950 to 2015).

Results: Thirty-two and 11 articles were included in the analysis of trends of diabetes prevalence and incidence, respectively. The prevalence and incidence of diabetes are increasing globally. A study of the trends on a finer geographic scale revealed contrasting results and confirmed a lack of trend data, notably in Eastern Europe and other low- and middle-income regions. Mortality is increasing in South and Central America and Africa, but is stabilising in the other regions of the world.

Conclusions: Trends in diabetes prevalence and incidence showed disparities among different groups of populations, which could be explained by disparities in lifestyle and access to medical facilities or could be related to the obesity epidemic. This study revealed important data gaps that have to be bridged to obtain a more complete and more accurate picture of the worldwide diabetes epidemic.
\end{abstract}

KEY WORDS: diabetes, incidence, prevalence, mortality, burden, global, disparities.

ADDRESS FOR CORRESPONDENCE: Maria Bota, International Prevention Research Institute (iPRI), 95 Cours Lafayette, 69006 Lyon, France, e-mail: maria.bota@i-pri.org

\section{INTRODUCTION}

Diabetes mellitus (DM) is a chronic condition mainly characterised by hyperglycaemia as a result of an insulin-related problem. Insulin is a hormone produced by the pancreas, and its role is to transport circulating glucose to the body cells where it is transformed into energy. When the pancreas does not produce enough insulin, or if the body cells fail to use insulin correctly, the circulating blood glucose levels increase and this is commonly known as DM (type 1 and type 2, respectively). If left untreated, DM may lead to the development of several complications: cardiovascular disease, neuropathy, nephropathy, and eye disease leading to retinopathy and blindness. Type 2 DM (T2DM) patients represent the vast majority of diabetic people in the world.

Quantifying the burden of DM is therefore a task of major significance for global public health. This was emphasised by the fact that DM was listed as one of the priority non-communicable diseases (NCDs) targeted for action by the United Nations [1].

Diabetes may be diagnosed through plasma glucose criteria, namely the fasting plasma glucose (FPG) or the two-hour plasma glucose (2-h PG) value obtained via a 75-g oral glucose tolerance test (OGTT), or the glycated haemoglobin (HbA1C) criterion. However, the diagnostic criteria are highly variable in time, and also from one country to another. In addition, there is a high rate 
of undiagnosed DM due to a large amount of the population never taking the tests.

A comprehensive analysis of the time trends of DM burden across the world is required to understand the complexity of the spread of the epidemic and the diversity of underlying causal factors.

The aim of the present article was to study the trends in DM prevalence, incidence, and mortality over time and by region of the world. Nine regions that would reflect the disparities in terms of DM prevalence and in terms of resource levels on a global scale were chosen as follows: Western Europe, Eastern Europe, Africa, the Middle East, Western and Central Asia, South-East Asia, Oceania, North America, and Central and South America.

\section{MATERIAL AND METHODS}

For the prevalence and incidence trends, a systematic literature review was performed. For the mortality analyses, data from the WHO mortality database were used [2].

\section{PREVALENCE AND INCIDENCE OF DIABETES}

\section{Literature search}

An online literature search was carried out in PubMed (January 1966-September 2019) for studies reporting data on the time trends of the prevalence and of the incidence of DM. The search was restricted to studies published in English, which contained the terms "diabetes", "prevalence"/"incidence", and "trends".

\section{Eligibility criteria and data extraction}

For each region, all observational studies reporting data on trends of prevalence/incidence were identified. If several articles reported data for the same country, the most recent study and/or the one with the largest number of points in time was selected. If there was no individual study for one region/country, then data from a systematic review or meta-analysis were considered. In order to be eligible, studies needed to have reported prevalence/incidence for at least two points in time and to have included data for the period from 2006 or later. Studies reporting on special populations, only on T1DM, or on gestational diabetes were excluded.

For the selected studies the following data were extracted: the first author's name, year of publication, country, study period, study design (population based, registry based, or clinic based), sample representation (national, regional, local, ethnic group), sample size, age of the studied population, DM diagnosis criteria (OGTT, FBG, HbAlc, medical record, or self-reported), overall prevalence/incidence with its corresponding $95 \%$ confidence interval and additional data by gender, or other subgroups if reported.

Data were extracted separately for men and women where possible. If reported, age-standardised prevalence/ incidence rates were prioritised over the crude rates.

\section{MORTALITY FROM DIABETES}

Diabetes mellitus was defined according to the International Classification of Disease (ICD) using the following codes: A063 or B020 in the seventh revision, A064 or B021 in the eighth revision, B181 in the ninth revision, and 1052 or E10-E14 in the $10^{\text {th }}$ revision.

These codes include the complications of DM including: ketoacidosis, renal complications, ophthalmic complications, or other complications. Diabetes was considered as a whole (T1DM and T2DM) because the mortality data were not available by type of DM in ICD7 to ICD9.

Diabetes deaths registered in the World Health Organisation (WHO) mortality database by November 2018 were extracted by gender and five-year age group for a selection of countries and for the longest period available (from 1950 to 2015). Population data were also extracted from the WHO database, unless they were missing, in which case published United Nations data were used instead.

Trends of DM mortality are presented by region. The selection of countries was made according to the number of years of available data and based on the quality of data [3]. Countries with low populations were not considered because their mortality rates fluctuate greatly over time. No low-quality data were used except for Africa, where too few data were available. Only countries with minimum 30 years of data and at least $90 \%$ of data coverage were used for analyses (except Africa and the Middle East, where too few data were available). Age-standardised mortality rates were computed for 100,000 person-years (PY) for each selected country and each gender using the Segi world population as reference. The trends over the last 10 years have been quantified using the annual percentage change (APC) using a linear regression of the log-transformed rates.

In order to compare the recent situations between different regions of the world, a mean mortality rate for the period 2011-2015 was computed for each selected country. If a country did not have any data on the period 2011-2015, the last year of available data was taken into account.

All analyses were performed for men and women separately.

\section{RESULTS}

Most of the studies reporting data on the prevalence of DM did not differentiate by DM type. However, because T2DM is much more frequent than T1DM, the prevalence reported hereafter concerns T1DM and T2DM together, but with T1DM representing only a very small contribution.

\section{PREVALENCE}

A total of 679 articles complied with the search criteria for trends in the prevalence of DM. Of these, 160 articles were selected after title and abstract reading, and 
then ordered by region. After full-text reading, 32 articles were selected for the analysis, covering 29 countries, plus five regions of Africa (prevalence data for Africa were only available by sub-region and not by country). All prevalence data are available in Appendix 1.

\section{Western Europe}

Six studies that reported prevalence trends of DM among countries from Western Europe were selected [4-9]. All six studies were representative of the national populations, and the data were either population or registry based. The highest rates were seen in Germany, with an increase from $3.1 \%$ in 1973 to $12.8 \%$ in 2006 among men and an increase from $3.5 \%$ to $12.4 \%$ in 2006 among women (based on self-reported DM) [4]. For the other countries, the prevalence trends were more homogenous, with increasing trends in all countries (Fig. 1).

\section{Eastern Europe}

Two studies were included from Eastern Europe, one from Greece, and one from Poland [10, 11]. In Poland, based on the number of patients who filled prescriptions,
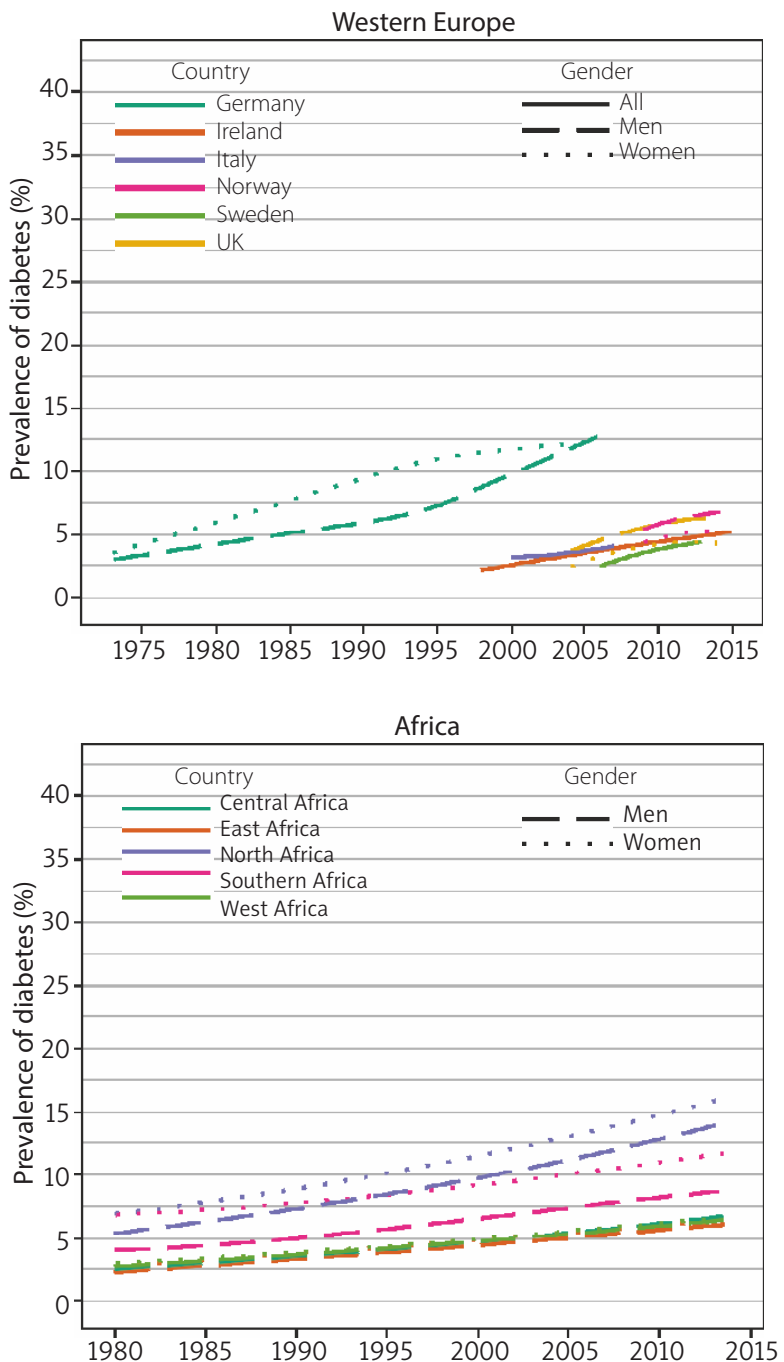

FIG. 1. Temporal trends in diabetes prevalence by region the prevalence of DM increased from 5.8\% in 2010 to $6.1 \%$ in 2014. Another study from Topor-Madry et al. [12] studied the prevalence of DM in Poland using data from different sources: 2013 all-billing records of the national insurance system, an epidemiological study, NATPOL, which involved the assessment of people with undiagnosed DM and the RECEPTOmetr Sequence study on prescriptions. This allowed the authors to assess separately the prevalence of diagnosed and undiagnosed DM: 2.17 million people who were aware that they had $\mathrm{DM}$ and 0.51 million who were unaware that they had $\mathrm{DM}$, resulting in an overall prevalence of $6.97 \%$. According to the WHO Diabetes country profiles, 2016, the prevalence of DM in Poland was 9.8\% in men, 9.3\% in women, and $9.5 \%$ overall.

The prevalence of DM in Greece increased from 8.5\% (in 2002) to $9.7 \%$ (2006) among men and from $7.8 \%$ (in 2002) to $9.3 \%$ among women (in 2006) (Fig. 1).

\section{Africa}

Trends in DM across Africa were extracted from the NCD Risk Factor Collaboration (NCD-RisC) - Africa
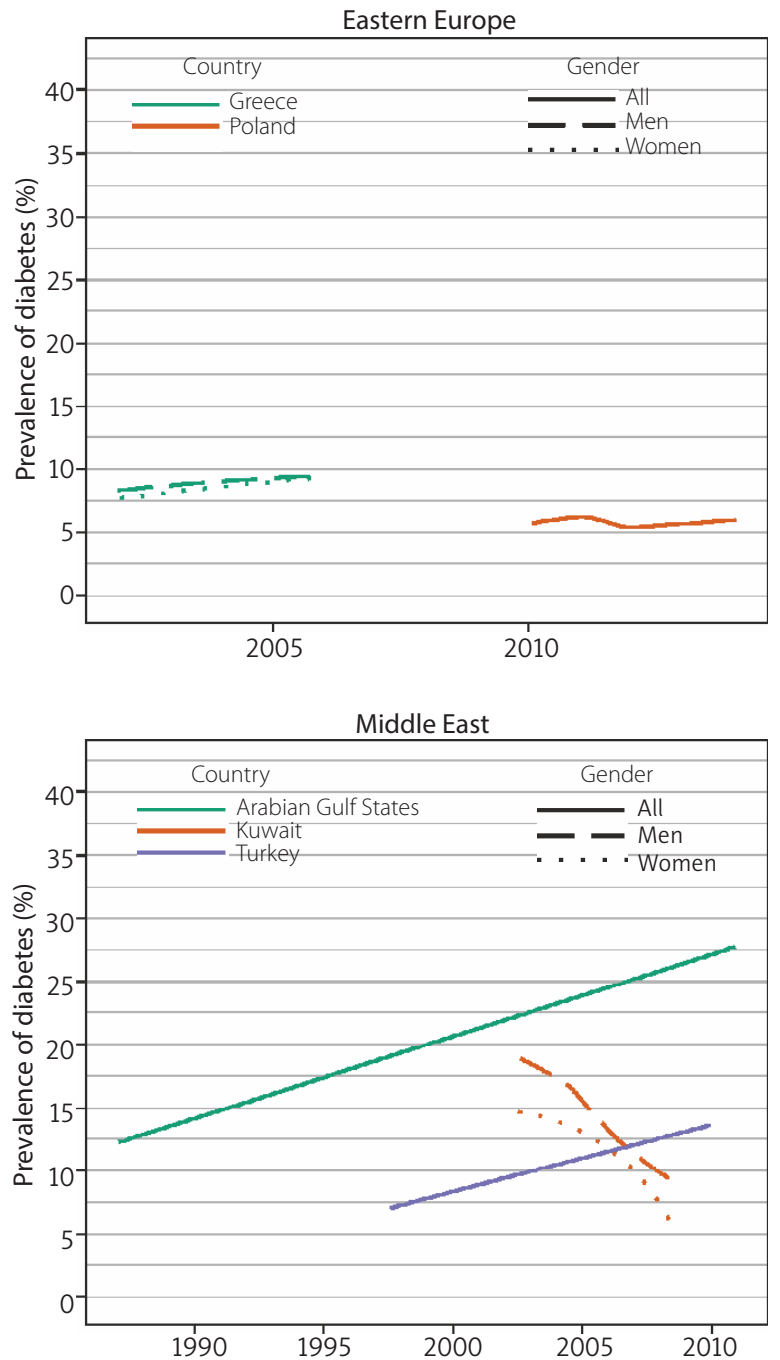

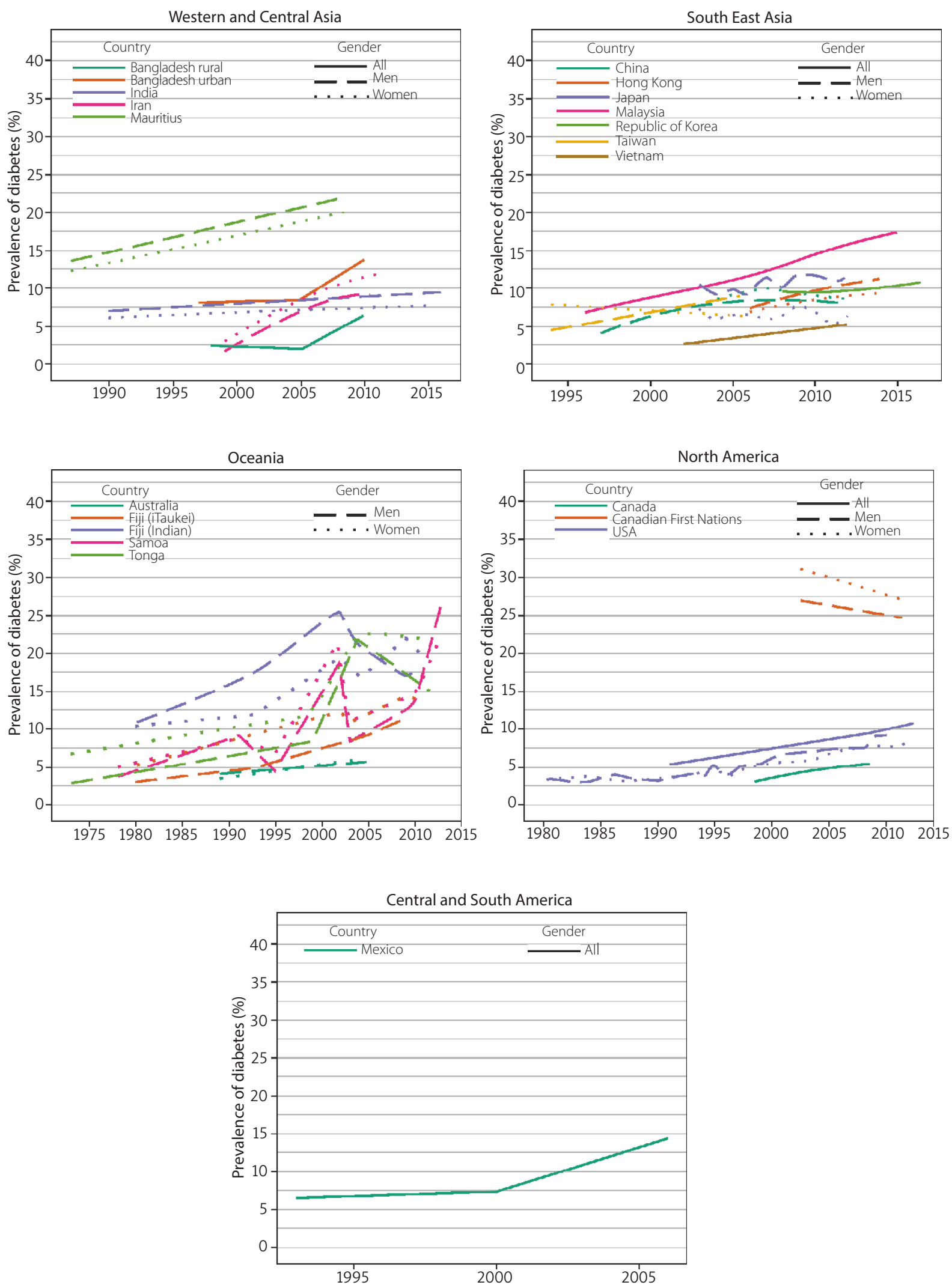

FIG. 1 Cont. Temporal trends in diabetes prevalence by region 
Working Group [13], which published an analysis of pooled population-based studies on the trends in obesity and DM across Africa from 1980 to 2014. Data came from 76 surveys (182,000 participants) for DM prevalence estimates (Tanzania, Tunisia, Cameroon, Egypt, and South Africa). The age-standardised prevalence of DM increased from $3.4 \%$ (95\% CI: $1.5 \%$; $6.3 \%$ ) to $8.5 \%$ $(6.5 \% ; 10.8 \%)$ in men, and from $4.1 \%(2.0 \% ; 7.5 \%)$ to $8.9 \%(6.9 \% ; 11.2 \%)$ in women. Estimates in Northern and Southern regions were higher than those in Central, Eastern, and Western regions (Fig. 1).

\section{Middle East}

Three studies were selected for the analysis of recent trends in DM prevalence in the Middle East [14-16]. These covered all Arabian Gulf States (Saudi Arabia, United Arabic Emirates, Bahrain, Oman, Kuwait, and Qatar), Kuwait in particular, and Turkey. In general, an increasing trend could be observed (Fig. 1). However, in Kuwait solely, Ahmed et al. [14] reported a decrease in the prevalence of DM from $19.1 \%$ (in 2002-2003) to $9.3 \%$ (in 2008-2009) among men and from $14.9 \%$ (in 2002-2003) to $6 \%$ (in 2008-2009) among women. The results were highly heterogeneous among studies.

One study [16] reported prevalence values by residential area: $17.0 \%$ (95\% CI: $16.4 \%$; $17.6 \%)$ for urban areas and $15.5 \%(14.8 \% ; 16.2 \%)$ for rural areas.

\section{Western and Central Asia}

Four studies reported trends in prevalence of DM [17-21] for four countries: Bangladesh, India, Iran, and Mauritius. Prevalence of DM was increasing steadily in all countries, both in women and in men as well as in rural and urban areas (Fig. 1). The highest prevalence was observed in Mauritius, with values reaching 22.3\% and $20.2 \%$ in 2009 in men and women, respectively [21]. In this study, subjects of South Asian ethnicity represented more than two thirds of the study population. Generally, the prevalence was higher in urban areas compared to rural areas.

\section{South-East Asia}

Seven publications reported data on trends in prevalence of DM in South-East Asia [22-28]. The highest values were observed in Malaysia (Fig. 1). In Japan and Korea the prevalence of DM was stable over the years, with values of around $11 \%$ in men and $7 \%$ in women. The lowest values were observed in Vietnam, but with a sharp increase from $2.7 \%$ in 2002 to $5.43 \%$ in 2012 .

\section{Oceania}

Several publications were found to report data on the trends of prevalence of DM in Oceania [29-32]. The prevalence of DM was very high among these populations, which are also subject to the epidemic of obesi- ty. The values for the prevalence of DM reached as high as $27 \%$ in Samoan men and $22.6 \%$ in Samoan women (Fig. 1). The trends were less pronounced in Australia.

\section{North America}

Three articles were selected for the description of prevalence trends in the USA and Canada [33-35]. Selvin et al. described the DM trends in a special population of the Canadian First Nations, who showed a very different pattern from the other populations: they used to have high prevalence rates, which are drastically decreasing. However, the majority of the North American population showed a steady increase in the prevalence of DM, with constantly higher values in the USA as compared to Canada (Fig. 1).

\section{Central and South America}

In Central and South America only one study fulfilled the selection criteria [36], which reported a rapidly increasing trend for the prevalence of DM in Mexico: from $6.7 \%$ in 1993 to $14.4 \%$ in 2006 (Fig. 1).

\section{INCIDENCE}

The literature search for trends in the incidence of DM yielded 769 results. Fifty articles were selected after title and abstract reading. Among these only 12 articles fulfilled the inclusion criteria and were used for the analysis. All incidence data are available in Appendix 2.

\section{Western Europe}

Six studies reported data on DM incidence trends in Western Europe [5-7, 37-39]. Recent DM incidence trends were quite similar among all countries, with stable trends over the last 10 years. The highest values were seen among Portuguese men while the lowest values were seen among British women (Fig. 2).

\section{Middle East}

One study reported data on recent trends in DM incidence and was conducted in Israel [40]. The incidence rates were decreasing constantly (Fig. 2).

\section{South-East Asia}

Two studies reported data on recent trends in DM incidence in South East Asia, one in Hong Kong [26] and one in Taiwan [41]. Incidence rates were higher in Hong Kong than in Taiwan, probably due to the westernisation of lifestyle in Hong Kong. However, the trends were constant over time in both countries, in men and women equally (Fig. 2).

\section{Oceania}

One study reported data on recent trends in DM incidence in Fiji [42], and it showed rates on a constant rise (from 261 per 100,000 in 1980 to 502 per 100,000 per year in 2011) (Fig. 2). 

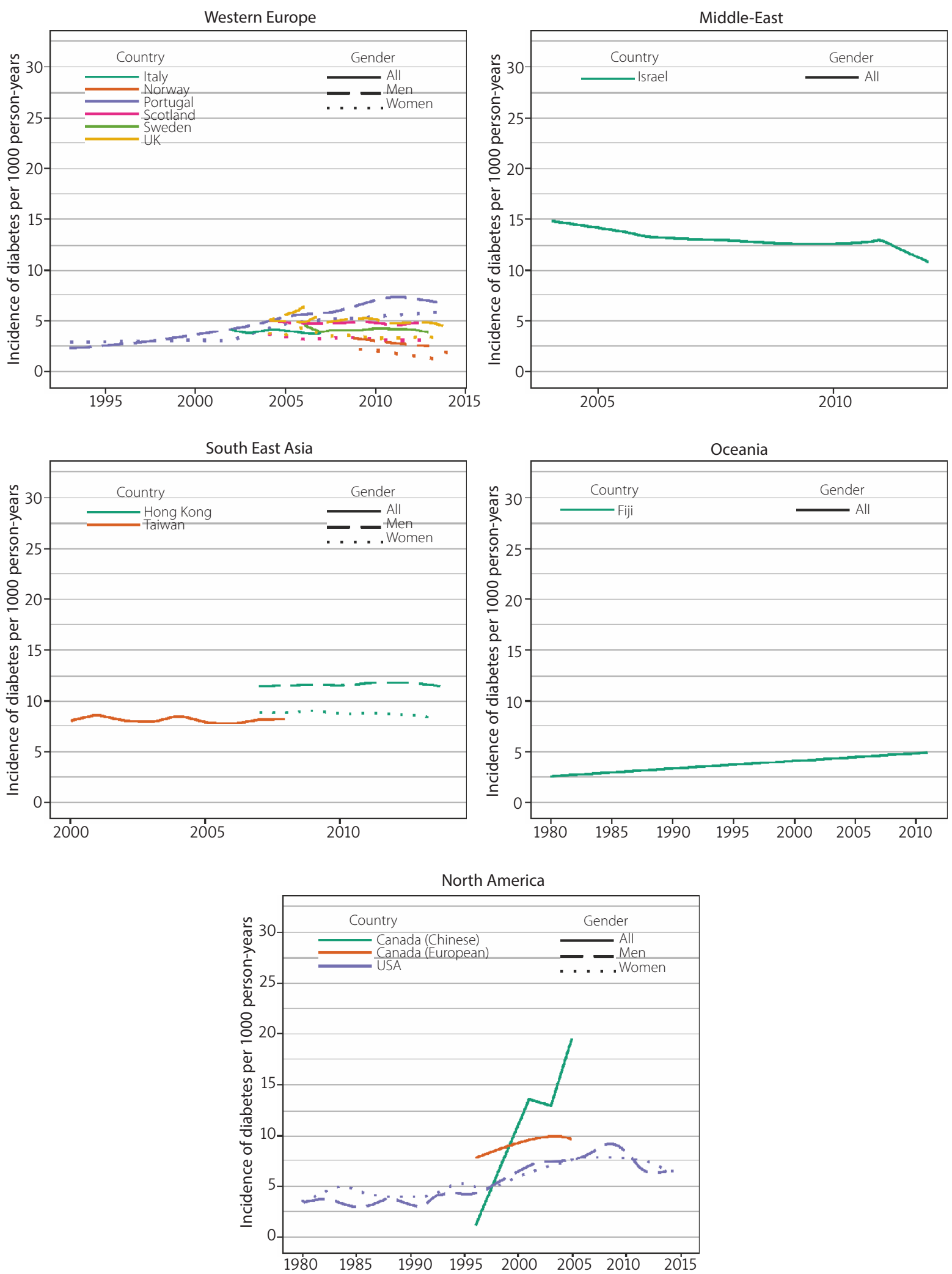

FIG. 2. Temporal trends in diabetes incidence by region

\section{North America}

Two studies reported data on recent trends in DM incidence in North America: one in Canada [43] and one in the USA [44]. In the USA, the trends were increasing up until 2010, after which they started to decrease until they reached around 650 new cases per 100,000 per year both in men and women in 2014-2015. Higher incidence rates were reported for European Canadians. Among 


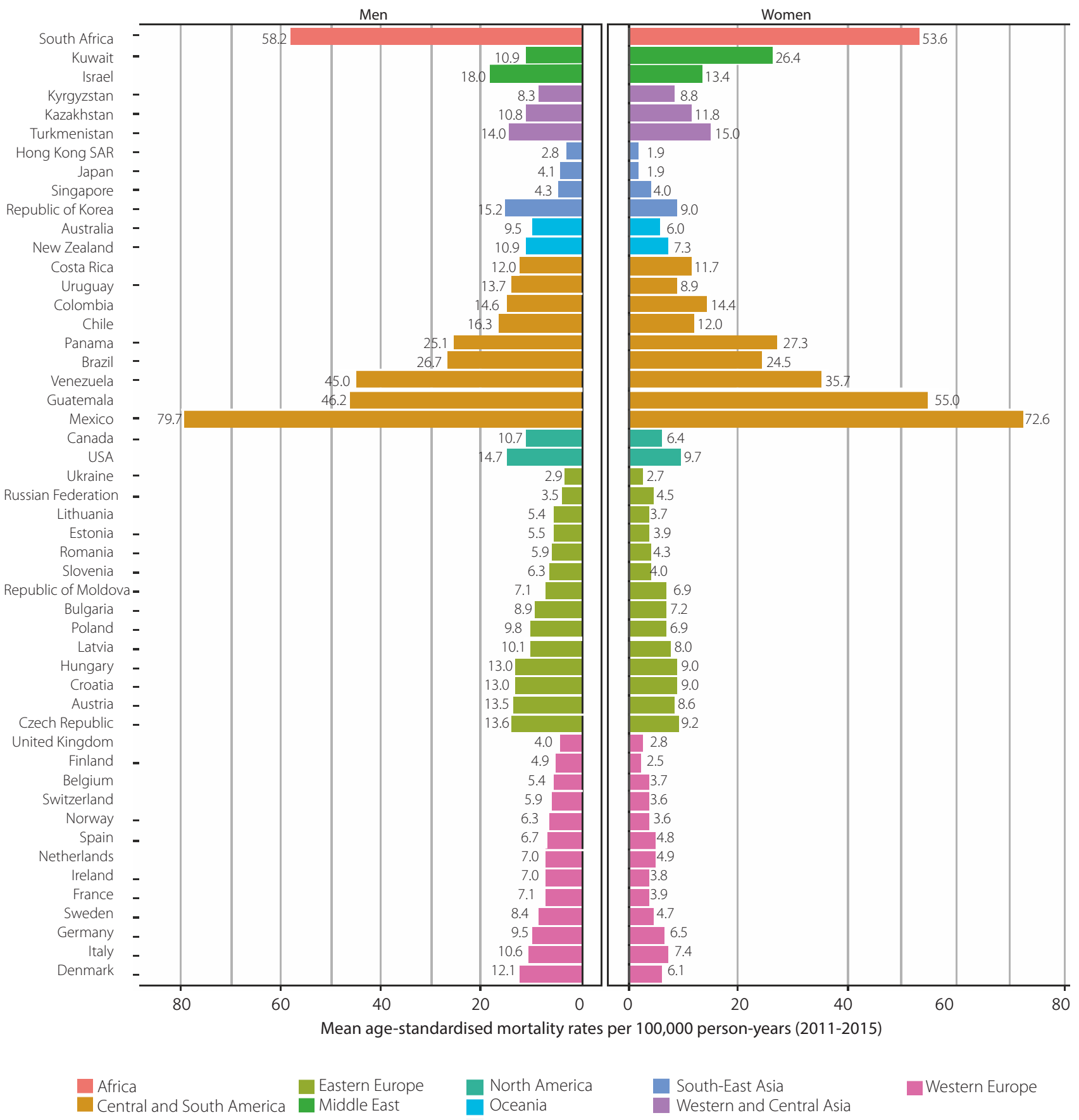

FIG. 3. Mean age-standardised diabetes mortality over the period 2011-2015 by country

Chinese Canadians the incidence rates showed a rapid growth from 130 per 100,000 per year in 1996 to 1960 per 100,000 per year in 2005 (Fig. 2).

\section{MORTALITY}

The mean mortality rates over the last years (20112015) are shown on Figure 3. Death rates from DM were higher in South and Central America, particularly in
Mexico and Guatemala and in South Africa, and lower in Europe, North America, and the Western Pacific.

\section{Western Europe}

Thirteen countries were selected to represent Western Europe: Belgium, Denmark, Finland, France, Germany, Ireland, Italy, the Netherlands, Norway, Spain, Sweden, Switzerland, and the UK. Diabetes mortality 
trends for men and women separately are presented in Figure 4 and Figure 5, respectively.

On average, mortality rates were slightly higher in men than in women. The trends seemed to decrease in women while in men the mortality rates remained stable over the period.

In women, over the period 2011-2015, the highest mean mortality was observed in Italy and in Germany with mean rates of 7.4 and 6.5 per 100,000 , respectively, while the lowest mean rates were observed in Finland and the UK, with mean mortality rates of 2.5 and 2.8 per 100,000 , respectively.

In men, the highest mean mortality was observed in Denmark and Italy, with mean rates of 12.1 and 10.6 per 100,000 , respectively, while the lowest mean rates were observed in the UK and Finland, with mean mortality rates of 4.0 and 4.9 per 100,000 , respectively.

\section{Eastern Europe}

Fourteen countries including the Russian Federation were selected to represent Eastern Europe: Austria, Bulgaria, Croatia, the Czech Republic, Estonia, Hungary, Latvia, Lithuania, Poland, the Republic of Moldavia, Romania, the Russian federation, Slovenia, and Ukraine. Diabetes mortality trends for men and women separately are presented in Figure 4 and 5, respectively. Globally, mortality rates were higher in men than in women. The trends appeared to increase from the 1950s to the 1990s and then stabilise or decrease for both genders.

Both in women and men, the highest recent upward trend was observed in the Czech Republic (APC $=5.54 \%$ for women and $\mathrm{APC}=6.82 \%$ for men), while the highest decrease was observed in Estonia (APC $=-8.62 \%$ for women and $\mathrm{APC}=-7.50 \%$ for men).

Over the last 10 years, in Poland, there was an increasing trend in mortality in men $(\mathrm{APC}=0.25 \%)$ and a decreasing trend in women $(\mathrm{APC}=-0.58 \%)$.

\section{Africa}

Diabetes mortality data were available only for South Africa and for the period 1993-2013 (Figs. 4 and 5). Over the period 1993-2015, DM mortality was slightly higher in women than in men. For the recent years (20112015), the mean mortality rate was 58.2 in men and 53.6 in women per 100,000 per year.

\section{Middle East}

Diabetes death data were only available in Israel and Kuwait for more than 20 years (Figs. 4 and 5). Mortality trends were similar in both genders with mortality rates slightly higher in women than in men in Kuwait. In Israel, the mean mortality was slightly higher in men than in women during the last 15 years.

In Israel and Kuwait, mortality trends varied greatly, with an increase in mortality from the 1970s to the 1990s, followed by a decrease until 2012-2015. For recent years (2011-2015) the mean mortality rate was 13.4 per 100,000 per year in women and 18.0 per 100,000 per year in men in Israel; and 26.4 per 100,000 per year in women and 10.9 per 100,000 per year in men in Kuwait.

\section{Western and Central Asia}

Data on DM deaths were available only for three countries: Kazakhstan, Kyrgyzstan, and Turkmenistan (Figs. 4 and 5). The trends were very similar among the three countries and among men and women, with an increase from the 1980s up until 1995, followed by a stable trend. The rates were highest in Turkmenistan both for men (mean of 14 per 100,000 per year during the period 2011-2015) and for women (mean of 15 per 100,000 per year during 2011-2015).

\section{South-East Asia}

Diabetes mortality data were available for four countries: Hong Kong, Japan, Republic of Korea, and Singapore (Figs. 4 and 5). Diabetes mortality rates were lower and more stable in Hong Kong and in Japan and higher and more variable in the Republic of Korea and Singapore. In all countries there was a decrease in the APC with the steepest decrease among Singaporean women $(\mathrm{APC}=-13.92 \%)$ and the lowest decrease among Japanese men $(\mathrm{APC}=-3.22 \%)$.

\section{Oceania}

Data on DM deaths were available only in Australia and New Zealand (Figs. 4 and 5). Both countries present similar trends, with higher mortality rates in New Zealand than in Australia, and a decreasing trend over the last 10 years both in men and in women.

\section{North America}

Diabetes deaths data were available for Canada and the USA (Figs. 4 and 5). From 1950 to 2010, mortality rates were slightly higher in the USA than in Canada for both genders. For both countries, mortality rates increased in men but decreased in women. In Canadian women there was a decrease of $3.71 \%$ in the APC over the last 10 years, while in US women the APC was slightly lower with only a $1.37 \%$ decrease. Similarly, in men there was a more significant decrease in the APC among Canadian men (APC $=-3.48 \%)$ versus the US men $(\mathrm{APC}=-0.33 \%)$.

\section{Central and South America}

Mortality data were selected for the nine following countries: Brazil, Chile, Colombia, Costa Rica, Guatemala, Mexico, Panama, Uruguay, and Venezuela (Figs. 4 and 5). A general tendency towards an increase in mortality rates was observed in South and Central America, with a stabilisation of mortality rates in a minority of countries such as Uruguay, Chile, Costa Rica, and Colombia.

The steepest increases in mortality rates were observed in Mexico and Guatemala. In the last 60 years 

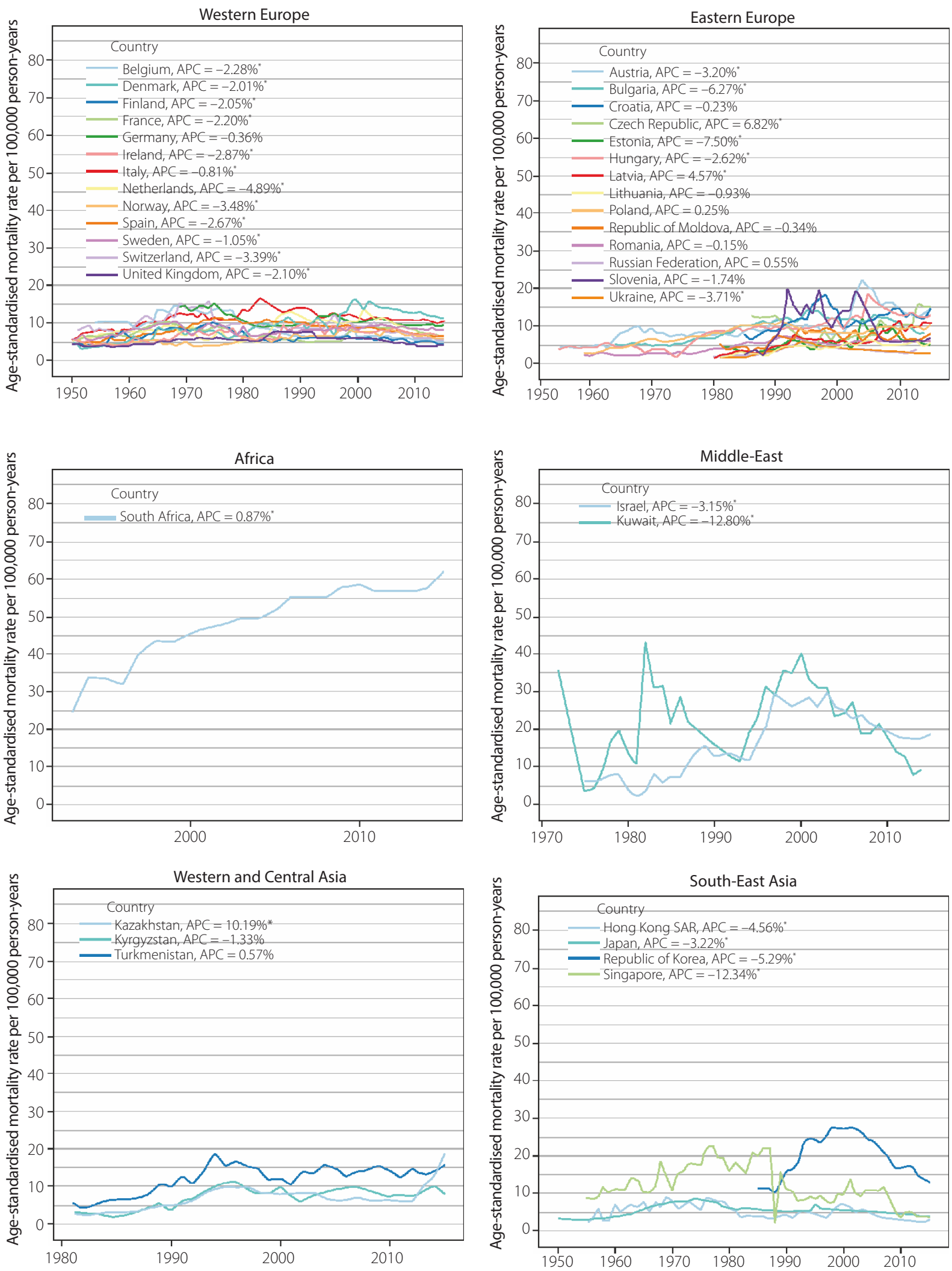

FIG. 4. Temporal trends in diabetes mortality by region in men 

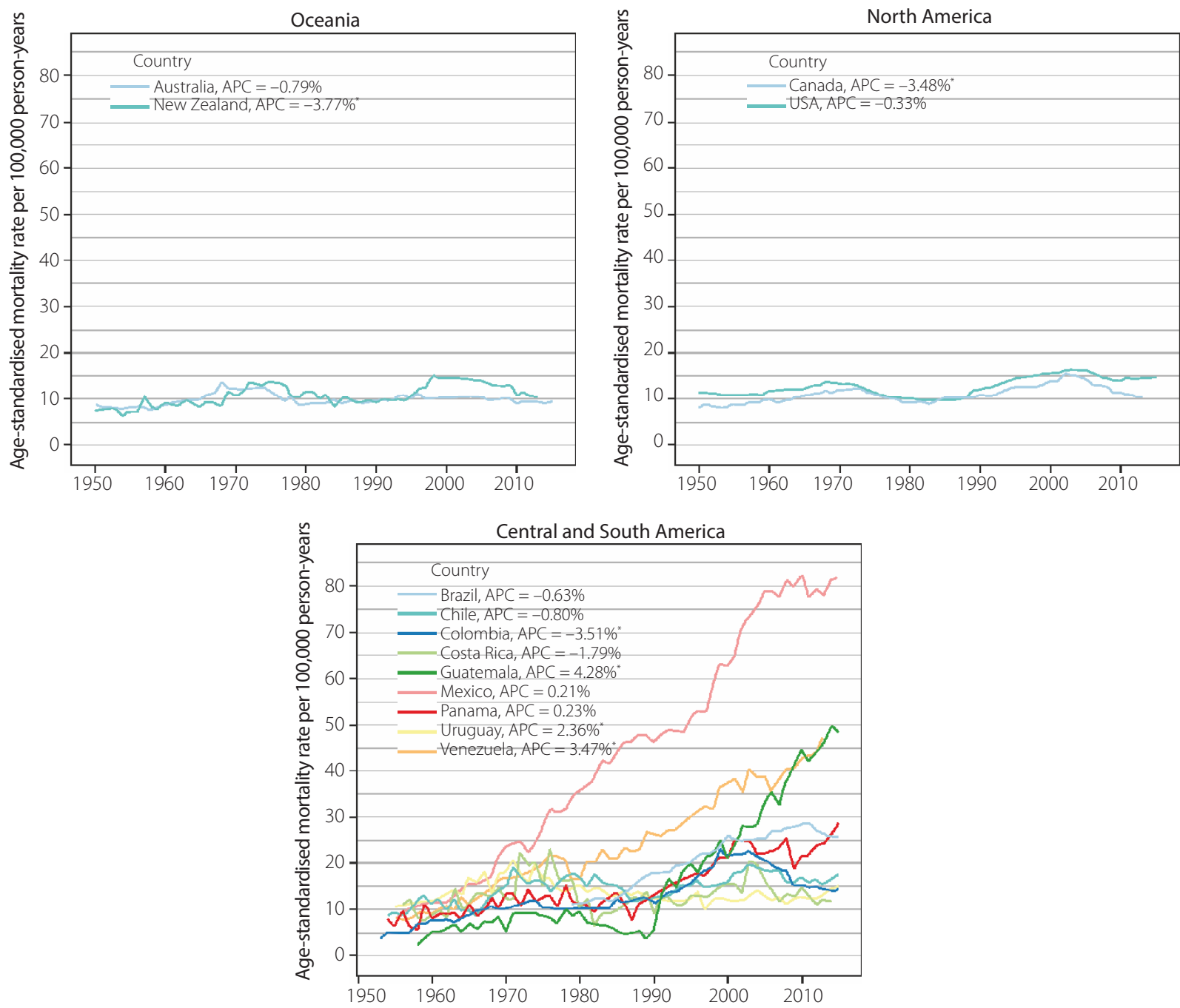

FIG. 4 Cont. Temporal trends in diabetes mortality by region in men

in Mexico, mortality rates showed an eight-fold increase among women and 10-fold among men. In recent years, mortality rates were the highest in Mexico (79.7 and 72.6 per 100,000 in men and women, respectively) and the lowest in Uruguay among women (8.9 per 100,000 per year) and in Costa Rica among men (12 per 100,000 per year). This represents significant disparity between the countries of South and Central America.

\section{DISCUSSION}

Compared to other reports on the descriptive epidemiology of DM, which focused on the current and future prevalence of DM, this study focused on the trends of the prevalence, incidence, and mortality over time.

Studies on DM prevalence around the world were very heterogeneous and gave heterogeneous results. Different study periods, geographical regions, population subsets, age ranges, and diagnosis methods hindered direct comparisons and quantitative, meta-analytic approaches. Diabetes prevalence tended to be higher in men than in women. Differences were observed for types of residential areas also, with higher prevalence in urban areas than in rural ones. We noticed particularly high values among certain populations, for instance in Western and Central Asia, the Middle East, Oceania, or among Native Canadians (as compared to the overall Canadian population).

Data on the trends of T2DM incidence were relatively scarce. Substantial variability was observed between the studies concerning the type of incidence reported (cumulative incidence, five-year incidence, etc.), the characteristics of the studied population (age, geographical localisation, ethnicity, etc.), the study period, the methodology, and DM diagnostic criteria. This heterogeneity made the results difficult to compare globally. However, in European and North American countries, where data is available, a tendency of stabilisation and/or decrease in T2DM incidence was observed in the last decade. More efforts should be made to collect data from regions where data is almost inexistent, such as South-East Asia, Africa, and the Middle East. 

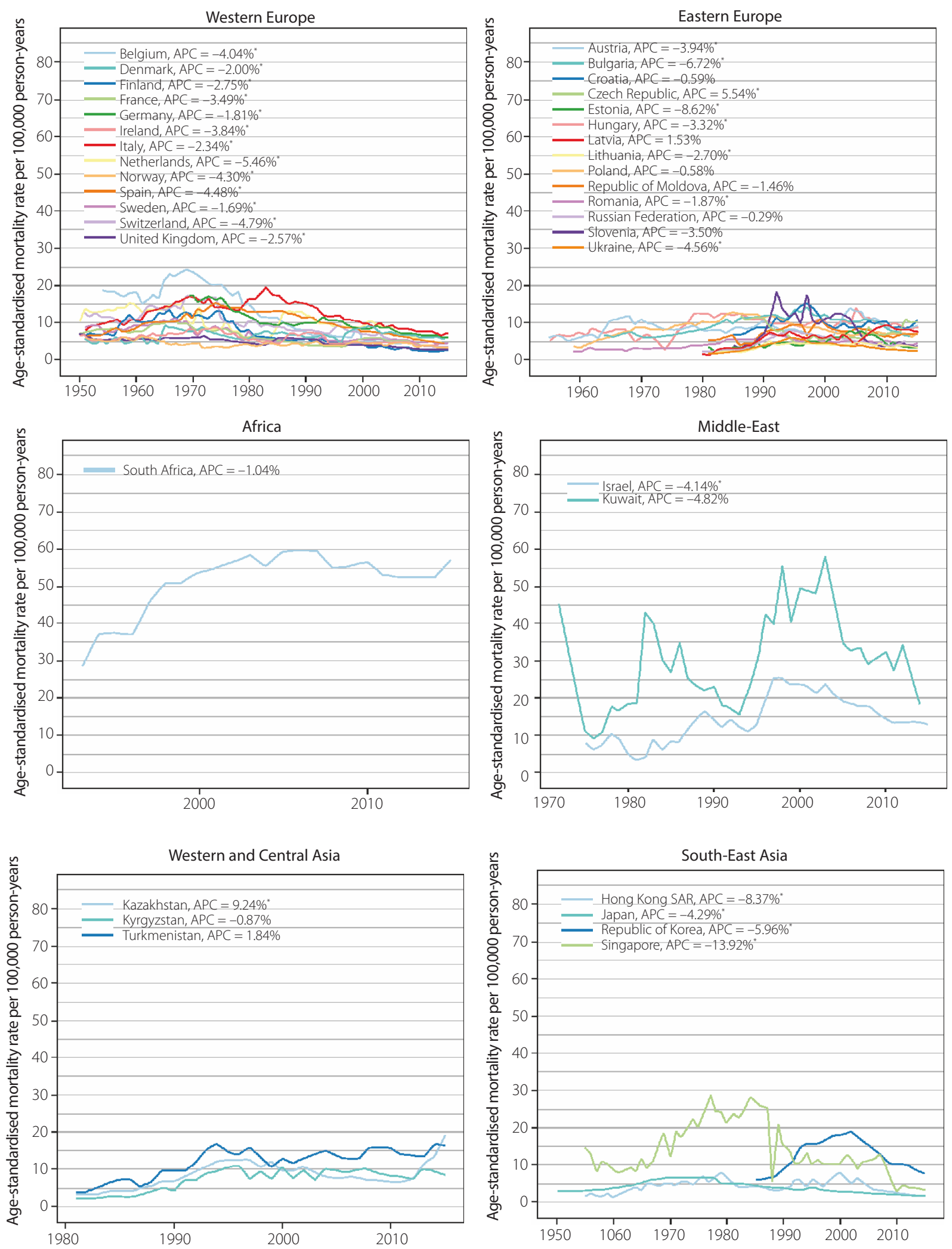

FIG. 5. Temporal trends in diabetes mortality by region in women 

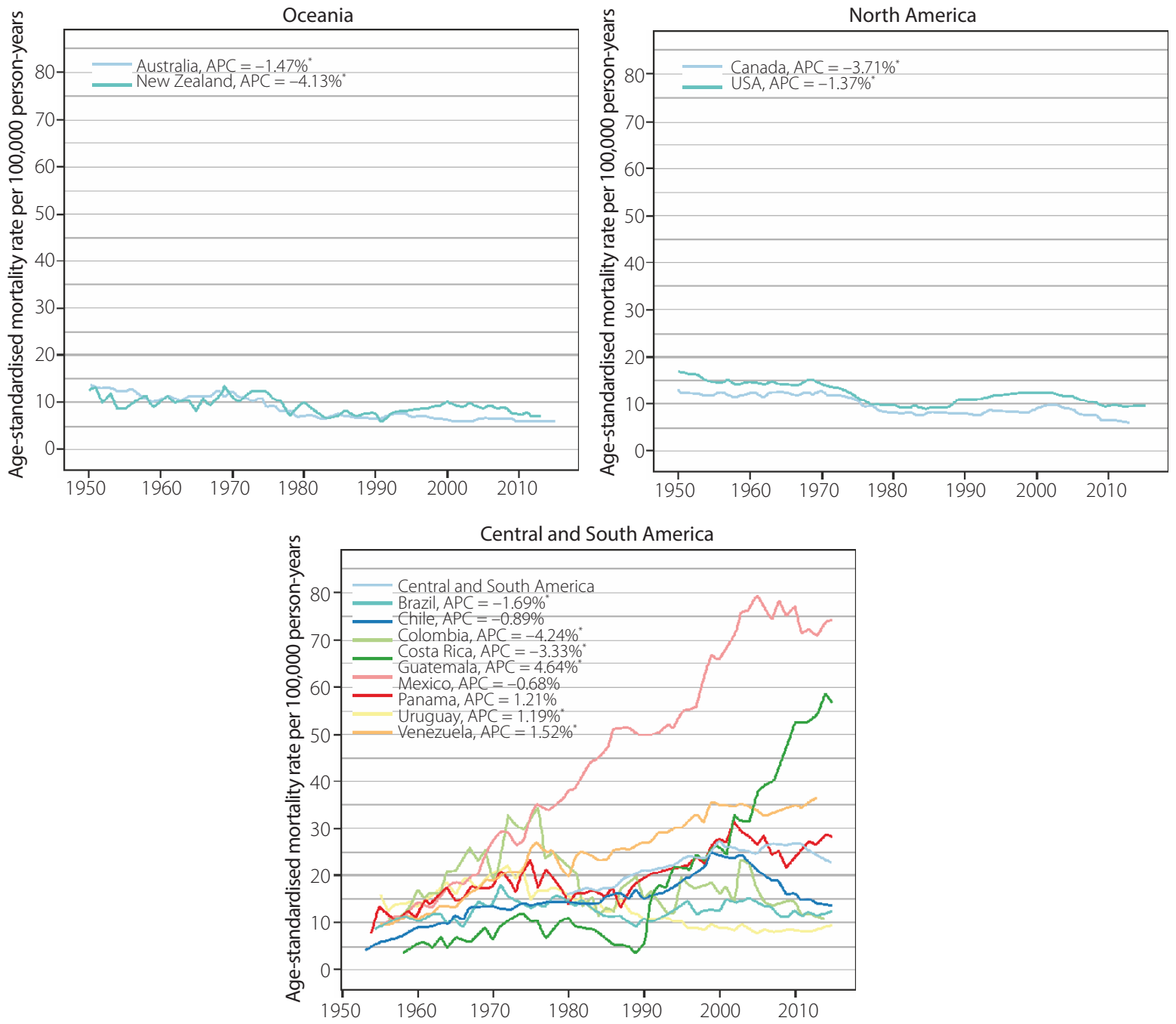

FIG. 5 Cont. Temporal trends in diabetes mortality by region in women

According to the WHO mortality database, deaths from DM were most frequent in South and Central America, particularly in Mexico and Guatemala, and in South Africa, and less frequent in Europe, North America, South East Asia, and Oceania. Overall, there was very little difference between men and women in the DM mortality rates, with slightly higher mortality rates in men than in women. In most of the regions, recent trends pointed towards a stabilisation of the rates or a decrease of the mortality rates except in South and Central America where the trends showed an increase.

These results were in line with other recent publications on several aspects of the burden of DM. In the "IDF Diabetes Atlas: Global estimates of DM prevalence for 2017 and projections for 2045" Cho et al. [45] produced estimates of the global burden of DM and its impact for 2017 and projections for 2045. For that, they performed a systematic literature review to identify published studies on the prevalence of DM and impaired glucose tolerance for the period 1990-2016. It was estimated that in 2017 there were 451 million (age 18-99 years) people with DM worldwide. These figures were expected to increase to 693 million by 2045. It was estimated that almost half of all people (49.7\%) living with DM are undiagnosed.

While DM is a serious disease affecting an ever-increasing number of people, the main morbidity and mortality arise from its complications. Hyperglycaemia associated with DM can lead to a number of disorders affecting the nerves, the heart and the blood vessels, the eyes, and kidneys. These are long-term complications that become more frequent with longer DM duration. There are also acute metabolic complications, associated with a lack of insulin treatment (ketoacidosis) or inappropriate blood glucose management (hypoglycaemia). In pregnancy, poorly controlled DM increases the risk of foetal death and other complications. All these complications increase the overall risk of dying prematurely and the burden of DM overall.

Another difficulty in the epidemic of DM is the undiagnosed cases. Selvin et al. [46] used the National Health 
and Nutrition Examination Survey results from 1988 to 1994 and from 1999 to 2014 in order to provide national estimates of undiagnosed DM for the USA. They found that in 1988 to 1994 , the percentage of total DM cases that were undiagnosed was $16.3 \%$, and from 2011 to 2014 this estimate had decreased to $10.9 \%$. Undiagnosed DM was more common in overweight or obese adults, older adults, racial/ethnic minorities, and persons lacking health insurance or access to health care. Determining the burden of undiagnosed DM is vital to monitoring public health efforts related to screening and diagnosis.

Moreover, some groups of people are affected by DM more than other groups. Differences in health status or access to health care among racial, ethnic, geographic, and socioeconomic groups are referred to as health disparities, and these can be noticed in the spread of DM epidemics.

This report showed disparities by region and by ethnicity. Despite rising trends in every region of the world, the heterogeneity of results made it difficult to further interpret the results.

Generally, further studies on the burden of DM are greatly needed in regions like Africa and Asia, where data is very scarce or non-existent. Special efforts must be made to collect more data in these regions in order to allow a more global view of DM incidence worldwide.

\section{CONCLUSIONS}

This article gives a broad overview of the burden of $\mathrm{DM}$ in the world. It showed great differences between, but also within, the main regions of the world. The highest burden of DM was seen in countries with low to middle income. Diabetes represents a great public health challenge for the future. The current trends suggest that low- to middle-income countries will have to deal with increasing numbers of diabetic patients in future years.

\section{DISCLOSURE}

The authors report no conflict of interest.

\section{References}

1. World Health Organization. Global report on diabetes 2016. Available from: https://apps.who.int/iris/bitstream/ handle/10665/204871/9789241565257_eng.pdf;jsession$\mathrm{id}=030329700 \mathrm{FB} 3 \mathrm{EF} 5 \mathrm{~A} 441 \mathrm{E} 48 \mathrm{C} 50 \mathrm{~A} 6 \mathrm{~F} 80 \mathrm{~F} 4$ ? sequence $=1$ (accessed: 19 September 2019).

2. World Health Organization. WHO mortality database. November 2018. Available from: http://www.who.int/healthinfo/statistics/mortality_rawdata/en/ (accessed: 19 September 2019).

3. Mathers CD, Fat DM, Inoue M, et al. Counting the dead and what they died from: an assessment of the global status of cause of death data. Bull World Health Organ 2005; 83 (3): 171-177.

4. Elkeles T, Beck D, Roding D, et al. Health and lifestyle in rural northeast Germany: the findings of a Rural Health Study from 1973, 1994, and 2008. Dtsch Arztebl Int 2012; 109 (16): 285-292.
5. Monesi L, Baviera M, Marzona I, et al. Prevalence, incidence and mortality of diagnosed diabetes: evidence from an Italian population-based study. Diabet Med 2012; 29 (3): 385-392.

6. Norhammar A, Bodegard J, Nystrom T, et al. Incidence, prevalence and mortality of type 2 diabetes requiring glucose-lowering treatment, and associated risks of cardiovascular complications: a nationwide study in Sweden, 2006-2013. Diabetologia 2016; 59 (8): 1692-1701.

7. Ruiz PLD, Stene LC, Bakken IJ, et al. Decreasing incidence of pharmacologically and non-pharmacologically treated type 2 diabetes in Norway: a nationwide study. Diabetologia 2018; 61 (11): 2310-2318.

8. Tracey ML, Gilmartin M, O’Neill K, et al. Epidemiology of diabetes and complications among adults in the Republic of Ireland 1998-2015: a systematic review and meta-analysis. BMC Public Health 2016; 16: 132.

9. Zghebi SS, Steinke DT, Rutter MK, et al. Comparative risk of major cardiovascular events associated with second-line antidiabetic treatments: a retrospective cohort study using UK primary care data linked to hospitalization and mortality records. Diabetes Obes Metab 2016; 18 (9): 916-924.

10. Gikas A, Sotiropoulos A, Panagiotakos D, et al. Rising prevalence of diabetes among Greek adults: findings from two consecutive surveys in the same target population. Diabetes Res Clin Pract 2008; 79 (2): 325-329.

11. Walicka M, Chlebus M, Brzozowska M, et al. Prevalence of diabetes in Poland in the years 2010-2014. Clin Diabetol 2015; 4 (6): 232-237.

12. Topor-Madry R, Wojtyniak B, Strojek K, et al. Prevalence of diabetes in Poland: A combined analysis of national databases. Diabet Med 2019; 36: 1209-1216.

13. NCD Risk Factor Collaboration (NCD-RisC) - Africa Working Group. Trends in obesity and diabetes across Africa from 1980 to 2014: An analysis of pooled population-based studies. Int J Epidemiol 2017; 46 (5): 1421-1432.

14. Ahmed F, Waslien C, Al-Sumaie MA, et al. Trends and risk factors of hyperglycemia and diabetes among Kuwaiti adults: National Nutrition Surveillance Data from 2002 to 2009. BMC Public Health 2013; 13: 103.

15. Alharbi NS, Almutari R, Jones S, et al. Trends in the prevalence of type 2 diabetes mellitus and obesity in the Arabian Gulf States: systematic review and meta-analysis. Diabetes Res Clin Pract 2014; 106 (2): e30-33.

16. Satman I, Omer B, Tutuncu Y, et al. Twelve-year trends in the prevalence and risk factors of diabetes and prediabetes in Turkish adults. Eur J Epidemiol 2013; 28 (8): 169-180.

17. Biswas $\mathrm{T}$, Islam A, Rawal LB, Islam SM. Increasing prevalence of diabetes in Bangladesh: a scoping review. Public Health 2016; 138: 4-11.

18. Esteghamati A, Aryan Z, Esteghamati A, Nakhjavani M. Differences in vitamin D concentration between metabolically healthy and unhealthy obese adults: Associations with inflammatory and cardiometabolic markers in 4391 subjects. Diabetes Metab 2014; 40 (5): 347-355.

19. Esteghamati A, Ashraf H, Khalilzadeh O, et al. Trends of diabetes according to body mass index levels in Iran: results of the 
national Surveys of Risk Factors of Non-Communicable Diseases (1999-2007). Diabet Med 2010; 27 (11): 1233-1240

20. India State-Level Disease Burden Initiative Diabetes Collaborators. The increasing burden of diabetes and variations among the states of India: The global burden of disease study 19902016. Lancet Glob Health 2018; 6 (12) e1352-e1362.

21. Magliano DJ, Söderberg S, Zimmet PZ, et al. Explaining the increase of diabetes prevalence and plasma glucose in Mauritius. Diabetes Care 2012; 35 (1): 87-91.

22. Chang $\mathrm{CH}$, Shau WY, Jiang YD, et al. Type 2 diabetes prevalence and incidence among adults in Taiwan during 1999-2004: a national health insurance data set study. Diabet Med 2010; 27 (6): 636-643

23. Han C, Zhang M, Luo X, et al. Secular trends in the prevalence of type 2 diabetes in adults in China from 1995 to 2014: A meta-analysis. J Diabetes 2017; 9 (5): 450-461.

24. Ikeda N, Nishi N, Noda H, Noda M. Trends in prevalence and management of diabetes and related vascular risks in Japanese adults: Japan National Health and Nutrition Surveys 20032012. Diabetes Res Clin Pract 2017; 127: 115-122.

25. Nguyen CT, Pham NM, Lee AH, Binns CW. Prevalence of and risk factors for type 2 diabetes mellitus in Vietnam: a systematic review. Asia Pac J Public Health 2015; 27 (6): 588-600.

26. Quan J, Li TK, Pang H, et al. Diabetes incidence and prevalence in Hong Kong, China during 2006-2014. Diabet Med 2017; 34 (7): 902-908.

27. Shin JY. Trends in the prevalence and management of diabetes in Korea: 2007-2017. Epidemiol Health 2019; 41: e2019029.

28. Tee ES, Yap RWK. Type 2 diabetes mellitus in Malaysia: current trends and risk factors. Eur J Clin Nutr 2017; 71 (7): 844-849.

29. Atlantis E, Lange K, Wittert GA. Chronic disease trends due to excess body weight in Australia. Obes Rev 2009; 10 (5): $543-$ 553.

30. Lin S, Hufanga S, Linhart C, et al. Diabetes and obesity trends in Tonga over 40 years. Asia Pac J Public Health 2016; 28 (6): 475-485.

31. Lin S, Naseri T, Linhart C, et al. Trends in diabetes and obesity in Samoa over 35 years, 1978-2013. Diabet Med 2017; 34 (5): 654-661.

32. Taylor R, Lin S, Linhart C, Morrell S. Overview of trends in cardiovascular and diabetes risk factors in Fiji. Ann Hum Biol 2018; 45 (3): 188-201

33. Geiss LS, Wang J, Cheng YJ, et al. Prevalence and incidence trends for diagnosed diabetes among adults aged 20 to 79 years, United States, 1980-2012. JAMA 2014; 312 (12): 1218-1226.

34. Riediger ND, Lix LM, Lukianchuk V, Bruce S. Trends in diabetes and cardiometabolic conditions in a Canadian First Nation community, 2002-2003 to 2011-2012. Prev Chronic Dis 2014 11: E198.

35. Selvin E, Parrinello CM, Sacks DB, Coresh J. Trends in prevalence and control of diabetes in the United States, 1988-1994 and 1999-2010. Ann Intern Med 2014; 160 (8): 517-525.

36. Villalpando S, Shamah-Levy T, Rojas R, Aguilar-Salinas CA Trends for type 2 diabetes and other cardiovascular risk factors in Mexico from 1993-2006. Salud Publica Mex 2010; 52 (Suppl 1): S72-79.
37. de Sousa-Uva M, Antunes L, Nunes B, et al. Trends in diabetes incidence from 1992 to 2015 and projections for 2024: A Portuguese General Practitioner's Network study. Prim Care Diabetes 2016; 10 (5): 329-333.

38. Read SH, Kerssens JJ, McAllister DA, et al. Trends in type 2 diabetes incidence and mortality in Scotland between 2004 and 2013. Diabetologia 2016; 59 (10): 2106-2113.

39. Zghebi SS, Steinke DT, Carr MJ, et al. Examining trends in type 2 diabetes incidence, prevalence and mortality in the UK between 2004 and 2014. Diabetes Obes Metab 2017; 19 (11): 1537-1545.

40. Karpati T, Cohen-Stavi CJ, Leibowitz M, et al. Towards a subsiding diabetes epidemic: trends from a large population-based study in Israel. Popul Health Metr 2014; 12 (1): 32.

41. Jiang YD, Chang $\mathrm{CH}$, Tai TY, et al. Incidence and prevalence rates of diabetes mellitus in Taiwan: analysis of the 2000-2009 Nationwide Health Insurance database. J Formos Med Assoc 2012; 111 (11): 599-604.

42. Morrell S, Lin S, Tukana I, et al. Diabetes incidence and projections from prevalence surveys in Fiji. Popul Health Metr 2016; 14: 45 .

43. Alangh A, Chiu M, Shah BR. Rapid increase in diabetes incidence among Chinese Canadians between 1996 and 2005. Diabetes Care 2013; 36 (10): 3015-3017.

44. Selvin E, Ali MK. Declines in the incidence of diabetes in the U.S. - real progress or artifact? Diabetes Care 2017; 40 (9): 1139-1143.

45. Cho NH, Shaw JE, Karuranga S, et al. IDF Diabetes Atlas: Global estimates of diabetes prevalence for 2017 and projections for 2045. Diabetes Res Clin Pract 2018; 138: 271-281.

46. Selvin E, Wang D, Lee AK, et al. Identifying trends in undiagnosed diabetes in U.S. adults by using a confirmatory definition: a cross-sectional study. Ann Intern Med 2017; 167 (11): 769-776.

\section{AUTHORS' CONTRIBUTIONS}

$M B, P B$ prepared research concept and design of the publication. $M B, A K, C P, M D$ collected data. MB, AK, CP, AM analysed data. $M B$ wrote the article. GG critically revised it. All authors contributed to the final version of publication. 